PÁGINA DEL EDITOR

\title{
Una metodología imprescindible para una eficiente investigación en el área de la salud
}

\author{
Ibeth Lady Villarroel Paredes', Alfredo Sejas Claros²
}

$\mathrm{E}$ n el área de la medicina como profesionales médicos debemos tomar la mejor acción ante un paciente, de ahí que las decisiones tienen que ser basadas en nuestros conocimientos e investigaciones de acuerdo a la patología que se presenta, pero no todas las ideas de los artículos publicados tienen la misma eficacia en la toma de decisiones en nuestro cotidiano desenlace y cada vez debemos afrontar el juicio y la experiencia propia agrupando la evidencia publicada o estudios realizados teniendo la certidumbre de que si la información es adecuada o confiable. Ambos criterios son imprescindibles al tomar la decisión correcta para obtener resultados satisfactorios, por tal motivo al verificar la confiabilidad debemos basarnos en niveles de evidencia científica que son sistemas de jerarquización que nos orientan a decidir el tipo de artículo publicado en el que podemos fundamentar de acuerdo al método de estudio empleado, por ello es de vital importancia la necesidad de evaluar la calidad de evidencia científica en cuanto a optar lo más propicio para el paciente, ello implica desarrollar una serie de juicios que se resume en buscar la diferencia o igualdad en el paciente con el artículo, tomando en cuenta una serie de factores que intrínsecamente se encuentran en los resultados comprobando así si hay significancia estadística y clínica llegando a justificar si se aplica a la persona tanto como las contraindicaciones y consecuencias, buscando certificar que el paciente obtenga el mismo resultado ${ }^{1,2}$.

La jerarquización de la evidencia de muestra la confiabilidad en el artículo; más simultáneamente existen riesgos de sesgos en la exploración de ese sistema como en la interpretación que uno hace de ella y en la aplicación, por lo tanto la búsqueda debe realizarse asociando la pregunta con la evidencia asegurando que responda de acuerdo a la necesidad individual y la realidad del paciente ${ }^{2}$.

Si confiamos que en que el sistema de evidencia sea la representación del estudio científico encontrado y que el mismo consiste en la verdad, podríamos confirmar que el nivel de evidencia está más cerca de la realidad y por lo tanto estos niveles nos exponen desde lo "muy recomendable" hasta "sin evidencia"1.

Este método científico también debe ser utilizado de acuerdo al lugar en el que nos encontramos tomando en cuenta la epidemiologia y el factor sociocultural.

En conclusión este sistema de evidencias nos permite guiar nuestras decisiones para dar la mejor alternativa para el paciente sin antes no guiarnos por nuestro razonamiento y asociación de la realidad del paciente con el estudio.

\section{${ }^{1}$ Ibeth lady Villarroel Paredes}

Editora en jefe Revista científica Ciencia Médica, Estudiante de quinto año de Medicina de la Facultad de Medicina-Universidad Mayor de San Simón, Cochabamba-Bolivia

${ }^{2}$ Alfredo Sejas Claros

Estudiante de segundo año de Medicina de la Facultad de Medicina-Universidad Mayor de San Simón, Cochabamba-Bolivia

Correspondencia a:

lady_ibeth@hotmail.com

Referencias:

1. Navas J, González J C, Mieth K. Niveles de evidencia, centro de gestión hospitalaria. Vía salud 2009; 48: 22-3. Disponible en: http://www.cgh. org.co/imagenes/calidad2.pdf

2.- Manterola C, Zavando M. Cómo interpretar los "Niveles de Evidencia" en los diferentes escenarios clínicos. Rev Chil Cir [revista en la Internet]. 2009 Dic [citado 2013 Dic 16]; 61(6): 582-595. Disponible en: http://www.scielo.cl/scielo.php?script=sci_arttexte'pid=S0718$40262009000600017 \mathrm{f} l \mathrm{lng}=\mathrm{es}$. http://dx.doi.org/10.4067/S0718-40262009000600017. 